\title{
Transforming Samples into Data - Experimental Design and Sample Preparation for Electron Microscopy
}

Alice F. Liang ${ }^{1 *}$, Chris Petzold ${ }^{1}$, Kristen Dancel-Manning ${ }^{1}$, Yolande Grobler ${ }^{2}$, Joseph Sall ${ }^{1}$, Chuxuan Zhou $^{3}$, Patrick H. Ren ${ }^{4}$ and Ruth Lehmann ${ }^{2}$

1. Microscopy Core Laboratory, DART, New York University Langone Health, New York, USA.

2. Howard Hughes Medical Institute, New York University Langone Health, New York, USA.

3. Concord Academy, Concord, Massachusetts, USA

4. Stuyvesant High School, New York, USA

* Corresponding author: Fengxia.liang@nyulangone.org

Recent advances in the field of electron microscopy (EM) have led to the development of technology and instrumentation, capable of achieving higher resolution than previously available. However, the development of procedures to obtain optimal preservation of specimens, remains a challenge. As a research driven microscopy core facility, our goal is to identify methods that offer superior preservation for a wide spectrum of samples through experimentation. These innovations, combined with the application of various advanced microscopy technologies, allow us to serve the interests of researchers from multiple disciplines, including material, chemical and biological science.

Cryofixation is widely accepted as a superior approach for immobilization and preservation of cellular architecture. Examples include high pressure freezing-freeze substitution for preservation of cells and tissues, and modern cryoEM technology for proteins and macromolecules. In addition to instrument limitations, not all samples are appropriate candidates for cryofixation. As such, conventional chemical fixation methods are still the most popular way to analyze the ultrastructural changes in experimental conditions. Vascular perfusion fixation is a special requirement for the structural preservation of liver, central neuron system, lung and muscle tissue. Immersion or bad perfusion fixation results in damage to cell ultrastructure, which sarcoplasmic reticulum (SR), T tubule and mitochondria in skeleton muscle are particularly susceptible to (Fig. 1).

$\mathrm{T}$ cells are specialized lymphocytes that play a central role in cell-mediated immunity, including antiviral immunity. Recent developments in CAR T-cell immunotherapy have added a new weapon in the fight against cancer. Mitochondria are metabolic organelles that actively transform their ultrastructure involved in antiviral responses, and cell death. Mitochondria fusion generates discrete and fragmented mitochondria that can increase the production of reactive oxygen species, facilitate mitophagy, accelerate cell proliferation and mediate apoptosis [1]. Also, mitochondria remodeling is a signaling mechanism that instructs T cell metabolic programming [2]. Morphological changes in mitochondria of T cells in cancer mouse models, is an important aspect for cancer research. However, the preservation of tiny amounts of flow sorted $\mathrm{T}$ cells from mice, is technical challenging. Traditional chemical fixation with glutaraldehyde and osmium tetroxide make it difficult to define clear mitochondrial structure in $\mathrm{T}$ cells, due to the small size of the cell with large nucleus (Fig. 2 top panel left). A modified rOTO protocol (rOTO: reduced osmium-thiocarbohydrzide-osmium), with additional uranyl acetate and lead aspartate staining, further increase heavy-metal content $[3,4,5]$, such that the membrane structure of $\mathrm{T}$ cell mitochondria is greatly highlighted (Fig. 2 top panel middle). This en bloc staining method can also provide contrast and increase sample conductivity, which reduces charging during SEM imaging (Fig. 2 top panel right). We also use a similar approach to study host cellular compartments that serve as Wolbachia niches. The rOTO protocol 
produces a clear outline of Wolbachia (Fig. 2, bottom panel middle), making it much easier to distinguish Wolbachia from other cytoplasmic organelles (Fig. 2, bottom panel middle red arrow) in cultured JW 18 cells, as compared to traditional TEM chemical fixation (Fig. 2 bottom left red arrow). This is also true for Wolbachia identification in the ovary of Drosophila. Serial block face scanning electron microscopy provides strong evidence that Wolbachia reside intracellularly, within modified host endoplasmic reticulum niches (Fig. 2, bottom right).

\section{References:}

[1] T Yu et al, Proc. Natl. Acad. Sci. USA, 103 (2006), p2653.

[2] DB Michael et al, Cell, 166 (2016), p.63.

[3] AM Seligman et al, J. Cell Biol., 30 (1966), p.424.

[4] T Deerinck et al, Microsc. Microanal., 16 (2010), p.1138.

[5] JC Tapia et al, Nat. Protoc., 16 (2012), p.193.
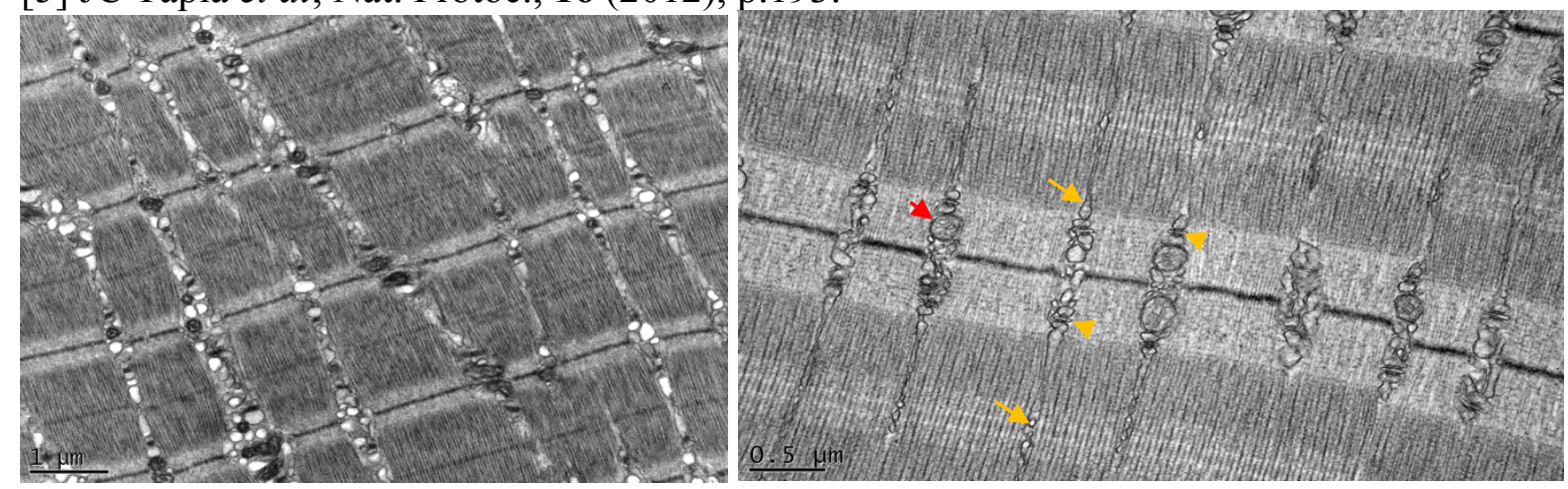

Figure 1. Perfusion fixation is very important for ultrastructural preservation of skeleton muscle. Left: immersion fixation; Right: perfusion fixation (orange arrow: SR; orange arrow head: T-tubule; red arrow: mitochondria).

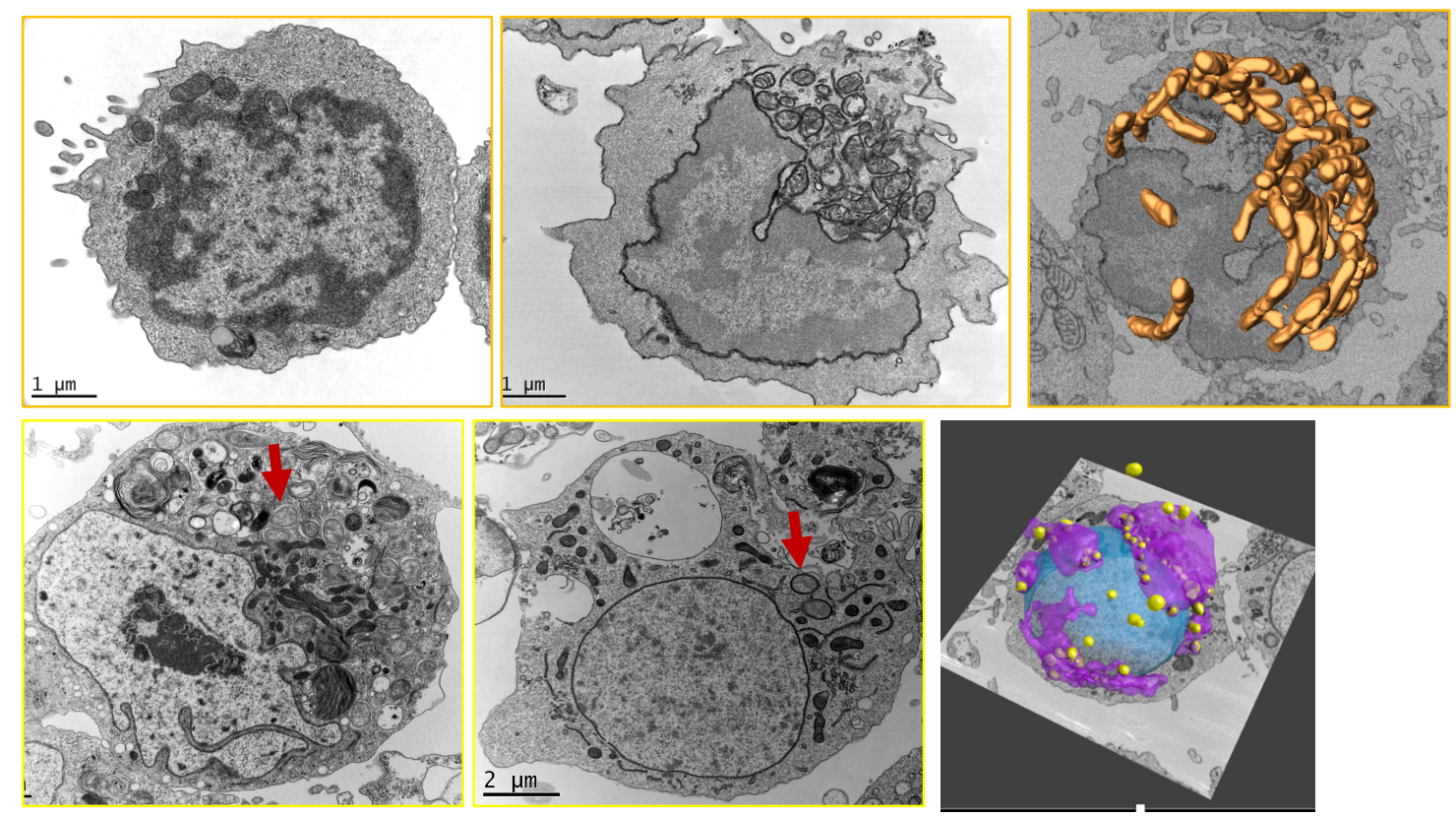

Figure 2. Different sample preparation method reveals different mitochondria (top panels) and wolbachia (bottom panels) appearance in flow sorted T cells (top panels), and cultured JW18 cells (bottom panels). 